\title{
Who Loves Virtue as much as He Loves Beauty?: Deep Learning based Estimator for Aesthetics of Portraits
}

\author{
Tobias Gerlach ${ }^{1, *}$, Michael Danner ${ }^{1,2, *}$, Le Ping Peng ${ }^{1,4}$, Aidas Kaminickas $^{2}, \mathrm{Wu} \mathrm{Fei}^{3}$ \\ and Matthias Rätsch ${ }^{1}$ \\ ${ }^{1}$ ViSiR, Reutlingen University, Reutlingen, Germany \\ ${ }^{2}$ Centre for Vision, Speech \& Signal Processing, University of Surrey, Guildford, U.K. \\ ${ }^{3}$ School of Computer Science, Xi'an Polytechnic University, Xi' an 710048, China \\ ${ }^{4}$ Philosophy, Hunan University of Science and Technology, Xiangtan, China \\ \{michael.danner, tobias.gerlach, le_ping.peng, matthias.raetsch\}@reutlingen-university.de, \\ aidas.kaminickas@student.reutlingen-university.de \\ ${ }^{*}$ T. Gerlach and M. Danner contributed equally to this work as first authors
}

Keywords: Benchmark Testing, Facial Databases, Attractiveness of Faces, Social Ethics, ELO Rating, Predictive Models, Deep Learning, Extreme-Gradient-Boosting Regressor, 3D Morphable Model.

\begin{abstract}
I have never seen one who loves virtue as much as he loves beauty," Confucius once said. If beauty is more important as goodness, it becomes clear why people invest so much effort in their first impression. The aesthetic of faces has many aspects and there is a strong correlation to all characteristics of humans, like age and gender. Often, research on aesthetics by social and ethic scientists lacks sufficient labelled data and the support of machine vision tools. In this position paper we propose the Aesthetic-Faces dataset, containing training data which is labelled by Chinese and German annotators. As a combination of three image subsets, the AF-dataset consists of European, Asian and African people. The research communities in machine learning, aesthetics and social ethics can benefit from our dataset and our toolbox. The toolbox provides many functions for machine learning with state-of-the-art CNNs and an Extreme-Gradient-Boosting regressor, but also 3D Morphable Model technologies for face shape evaluation and we discuss how to train an aesthetic estimator considering culture and ethics.
\end{abstract}

\section{INTRODUCTION}

\subsection{Motivation}

"In the post-Cold War world, the most important distinctions among peoples are not ideological, political, or economic. They are cultural." (Salzborn and Stich, 2016). Culture is the wealth created by mankind. Since human beings exist and develop in regions, countries, and nations, culture must present diversity, and each culture is different.

From a philosophical point of view: Chinese aesthetics and Western aesthetics are the aesthetics of two different ideological and cultural systems. The traditional Chinese aesthetics represented by Confucius (Wang, 2016) and Laozi (Kelly, 1998) are experience, ethical, and social philosophies. In contrast, Western aesthetics represented by Plato (Hyland, 2008) and Aristotle (Porter, 2017) are rational, religious, and psychological philosophies.
The standard of aesthetics depends on different cultures. For example, Book of Poetry, the oldest collection of folk poems in China, was written from the $11^{\text {th }}$ century BC to the $6^{\text {th }}$ century BC, reflecting people's thoughts and feelings, life style and understanding of natural phenomena in that era. It describes the beauty of an image as: "Her fingers like soft blades of reed, like larva white her neck is slender" (Yuanchong, 1993). Although time is changing, Asians still prefer to have white skin and a, bright-eyed look with open eyes (Sturm et al., 2010).

An Asian with an umbrella is a common sight in summer to provide protection from being sun-tanned. "White skin" has emerged as a central desideratum of consumer culture in affluent Asia (Sahay and Piran, 1997; Li et al., 2008), but from the study of Western cultural history and film and television works, it is found that American skin beauty advertisements tend to use models with a tanned or darker skin tone rather than a pale complexion as beauty (Xie and 
Zhang, 2013). Jones et al. (Jones et al., 2000) examined United Kingdom college students and found that the majority reported engaging in and enjoying sunbathing.

China has a proverb: "Loving beauty is part of human nature". No matter where you're from, no matter what aesthetic standards you have, the yearning for beauty is the same because aesthetics has affected many aspects of peoples' lives. Various evidences have shown that facial attractiveness plays a key role in a variety of judgements in the course of social interaction (Little and Roberts, 2012; van der Geld et al., 2007; Langlois et al., 2000; Anderson et al., 2008), attractive people are even more likely to be hired (Aharon et al., 2001).

It should be noted that this study is not based on a male-centred culture and uses male positions, concepts, and perspectives to measure and assess the value of women. In fact, we are gradually enriching the proportion of male photos in the photo library which will extend our dataset and further study will be published later. Because we label pairwise, for annotators it is too hard to rank attractiveness in one pair with a male and female face. The purpose of this work is to compare current East and West objective aesthetic standards to better understand the aesthetic needs of different cultures and to provide an aesthetic tool for cross-cultural communication, international business, and other fields and avoid cultural discrimination or conflict.

\subsection{Literature Research}

(Xie et al., 2015) published a database, containing 500 images with aesthetics ratings: "SCUT-FBP: A Benchmark Dataset for Facial Beauty Perception". They not only built the database but also trained different models for assigning aesthetic values automatically. Main take away of this work is a dataset for Asian subjects and the indication, that Deep Learning approaches give the best results for that kind of task. We will not only improve on the choice of the network but also improve the data preparation. SCUTFBP only used 400 images for training without any augmentations. The network they used was a 6 layered Convolutional Neural Network.

(Eisenthal et al., 2006) also describe a machine learning approach for beauty ratings which shows an objective concept of beauty and its origins very well. They also used a machine learning approach, but only had 184 images for training, which is a very small number in machine learning. They used support vector machines and Neural Networks, which rely on features, selected by the programmer. We will use
Convolutional Neural Networks to select features automatically.

(Redi et al., 2015) focuses on the quality of digital portraits. They only used manually extracted features for regression upon which we will improve.

\subsection{Main Contribution}

Research into aesthetic estimation using the latest machine learning tools is a technological challenge that psychologists and computer scientists have recently addressed, and there is still a lot of potential for improvement. The results of this work should help scientists in the future to combine aesthetic aspects with artificial intelligence.

Aesthetic-Faces-dataset (AF-dataset): First of all, we provide to the best of our knowledge the biggest dataset with currently 12.684 .492 aesthetic score annotations for the machine learning, aesthetics and social ethics research communities. This database is under continuous development and will be published on GitLab. Currently, the database consists of three subsets of images: Olympic sports athletes, celebrities and a dataset containing half Asian, half Caucasian faces (together 5.484 subject faces). We provide the aesthetic scores, but also several other metadata, like age, gender, eyeglass wearer, sport, ethnic group and other characteristics of the subject faces and also meta-data of the over 1,000 annotators with different gender, age and cultural origin.

Aesthetics Estimation Toolbox: Additionally, we develop and release a toolbox for researchers in social ethics to analyse or synthesise faces and prove their assumptions in ethical, legal and social implications (ELSI). This paper includes a baseline approach for an aesthetics score estimator. But the toolbox is also interesting for the machine learning and face modelling community. 3D Morphable Model technology is provided for deeper research and for regression and we will use standard and state-of-theart approaches. Additionally, a comparison of machine learning tools like Convolutional Neural Networks and Extreme Gradient Boosting is projected.

Social Ethics Correlations: Finally, we describe and analyse first correlations and social ethic aspects within the AF-dataset based on the integration of artificial intelligence technologies into the practice of social research. 


\section{METHODOLOGY}

\subsection{Machine Learning Approaches}

We have been researching aesthetics estimation since 2014 and published some work about aesthetic evaluation of face images on CNN trained networks (Fei et al., 2019). By changing the image background for training, we could improve the overall performance and show that the network benefits from different backgrounds to prevent overfitting and increase accuracy. Further improvements have been achieved by switching to state-of-the-art regression methods.

Further improvements on the architecture and data preparation of the $\mathrm{CNN}$ approach are currently in development and will be published. The LeNet and F-Net approaches achieve first promising results but provide too few features and layers to generate accurate results. Therefore, we will repeat our experiments with state-of-the-art networks from (Russakovsky et al., 2015), change the output layers fitting for regression, and evaluate how they perform.

We will also test extreme gradient boosting, since many Kaggle competitions are won by applying the XG-Boost regressor (Chen and Guestrin, 2016).

Kaggle is a commonly used homepage for providing databases and data science competitions.

\subsection{D Morphable Models}

Using 3D Morphable Models to analyse human face is a popular approach to face analysis. Characteristic for Morphable Models is to generate a 3D shape and its colour separately. We will concatenate the AF-dataset with 3D face shapes and analyse geometric proportions of the mouth, nose, eyes and chin in 3D space to aesthetic labels. The dataset will then also be classified with typical face types like oval, triangle, diamond, rectangle, round, heart and oblong.

\subsection{XG-Boost Regressor}

We will also use "Extreme Gradient Boosting", an MatLab implementation of Gradient Boosting (Friedman, 1999) algorithm. This algorithm was first published by Friedmann, who might have the same influence on machine learning as Rosenblatt has with the perceptron (Rosenblatt, 1958). Although this algorithm is very old in the fast-evolving machine learning world, many competitive regression tasks are still won with XG-Boost regressor.

\section{AESTHETIC-FACES DATASET (AF-DATASET)}

Every AI's solution to a problem begins with preparing a dataset. To investigate student surveys for rating the aesthetics of women's faces it was necessary to prepare datasets with several thousands of images. Our research has already taken 6 years and in this time, we have investigated 12 surveys with 4 different datasets. In 2013 we started with the dataset that we call the Olympics-subset. Since 2016 we have been working on celebrity images and this year we first started with SCUT-FBP pictures. None of the images are normalized (except SCUT-FBP dataset), which means we rate the beauty perception of the image themselves, rather than the beauty of the females faces contained in the images. Below we will briefly discuss all above-mentioned datasets, but we will pay more attention to our recent surveys based on celebrities and SCUT-FBP images. Dataset analysis was started exclusively for this proposal paper. The resulting database will contain a mixture of all the researched datasets with aesthetic correlated labels.

For the annotators, we used different datasets and evaluation methods. Data labels were acquired by annotating images from Celebrities- and Olympicsubsets. The datasets were split into two halves which had to compete against each other. The student has to select either the left or the right image to find the more aesthetic person (see figure 1). This has several advantages over the direct rating of the images as mostly described in (Xie et al., 2015).

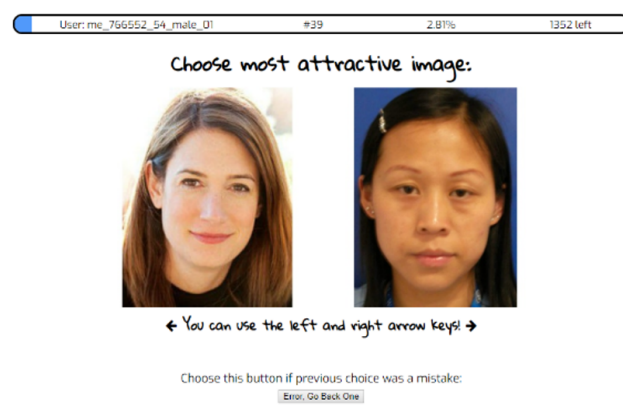

Figure 1: One out of 1352 annotation screens to choose either the left or right image on the Asian-Caucasian-subset.

The ratings of all students were saved to a text file and then evaluated. Starting this year, we have been using the ELO-rating (Lehmann and Wohlrabe, 2017) to replace mean calculations. 


\subsection{Olympics-subset}

The Olympics-subset (International Olympic Committee, 2013) consists of 1156 images of female Olympic athletes. Every image is labelled with player age, country of origin, height, weight, and sports art. In 2014, 322 students participated in our survey. Based on the survey output every image was rated with a score value ranging from 0 to 1 . In Figure 2 the average attractiveness is mapped on an Olympic sport. According to this distribution, the most aesthetic female athletes are figure-skaters with an average score of 0.6 in opposite to speed-skating athletes with a 0.45 ranking.

In Figure 3 we show how attractiveness depends on the age of Olympics players. As expected, the most attractive are females from 18 to 30 years old.

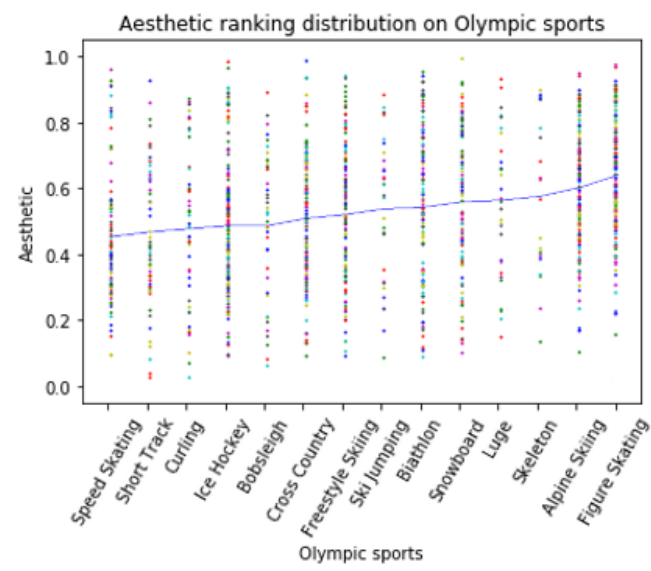

Figure 2: Aesthetic ranking distribution on Olympic sports. Every dot equates to a subject's image.

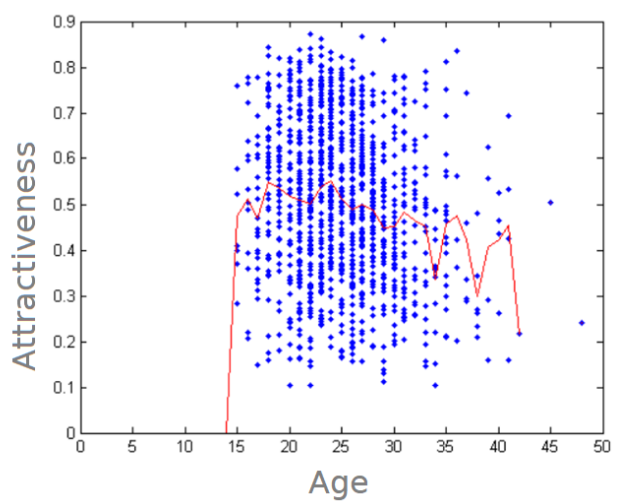

Figure 3: Attractiveness vs. age. Red line shows average values, every blue dot equates to a subject's image.

Various evidences have shown that faces that appear older are less attractive (Deffenbacher et al., 1998; Henss, 2006). Cigarette ads with young persons were found to appear more often in magazines with younger audiences and for menthol brands. Re- gardless of viewer age, younger models were judged as more attractive than older models (Mazis et al., 1992). From this study on large datasets we demonstrate that if the age increases, the attractiveness curve gradually declines; the correlation between age and beauty is significant even in all subsets across very different cultures of the subjects but also across different cultures of annotators (see Figure 10, Figure 11 and Figure 6). It should be noted that the attractiveness curve is relatively linear from 20 to 35 and on some subsets even till 60 years old subjects. For low and high ages, the data are not trustworthy, because there are only a few examples.

\subsection{Celebrities-subset}

In 2016 we have started to create a new dataset by creating a subset of Labelled Faces in the Wild, abbreviated LFW (Huang et al., 2007) that is a public benchmark for face verification also known as pair matching. The LFW dataset consists of 13,233 pictures of 5,749 popular actors, singers or politicians. These images were sorted out from male faces by our researchers. Afterwards, image information like age, ethnicity, and glasses (yes/no) was generated and the new image set with 1,578 pictures is now called the Celebrities-subset.

Figure 4 shows the distribution of age in this dataset. We can see a Gaussian distribution with a mean value of 43 years. The majority of the celebrities didn't wear glasses as seen in Figure 5. The same figure also shows that around 1300 are white, 200 black and the rest Asian females faces. Our work concatenates aesthetic labels with characteristic attributes; for example Figure 6 shows the evaluation of aesthetic labels and subject's ages.

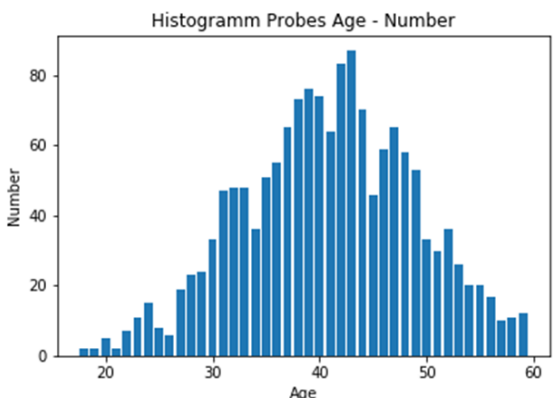

Figure 4: Distribution of age.

Currently, 171 students from different cultures participated in annotating the Asian-Caucasiansubset. The annotation outcome was calculated by using both the direct ranking that counts the up- and down-votes and the ELO-rating system for calculating relative numbers. 


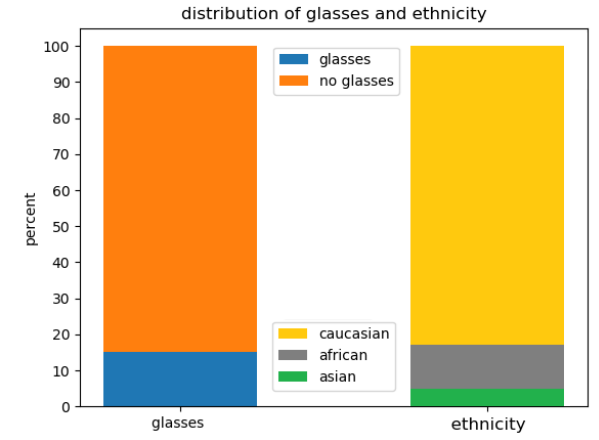

Figure 5: Glasses and ethnicity distribution on the Celebrities-subset.

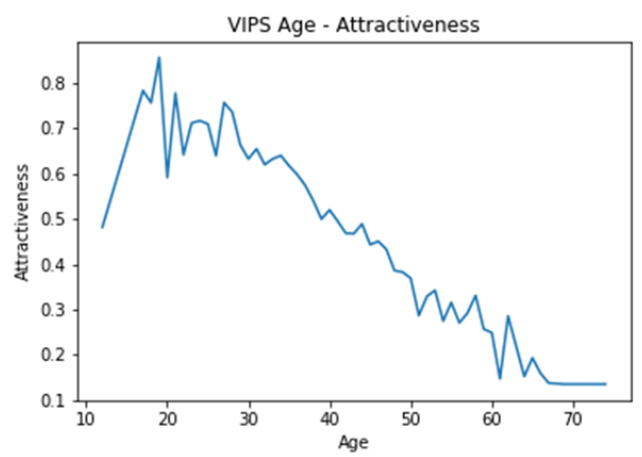

Figure 6: Correlation of age and attractiveness on the Celebrities-subset.

Figure 7 shows a histogram about ethnicity and attractiveness relation. The result here depends a lot on survey participants that will be discussed in more detail later on. It can also be concluded that people without glasses are more attractive.

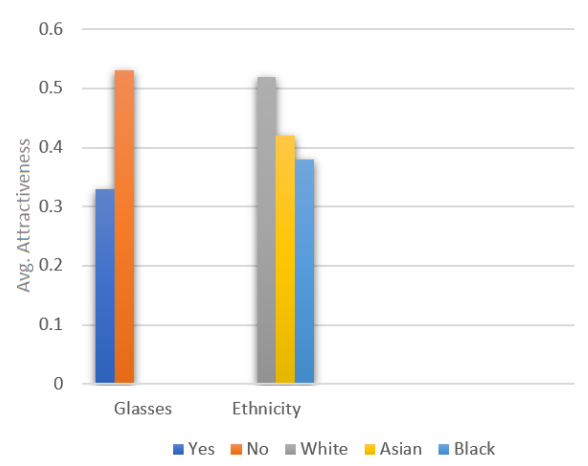

Figure 7: Celebrities-subset glasses and ethnicity vs. attractiveness.

\subsection{Asian-Caucasian-subset}

This year we started investigating surveys on a diverse benchmark database for multi-paradigm facial beauty prediction as released by the Human-Computer Intelligent Interaction Lab of South China Univer- sity of Technology and called SCUT-FBP5500database (Liang et al., 2018). The SCUT-FBP dataset has 5500 frontal faces which are categorized in Asian male, Asian female, Caucasian male and Caucasian female. The dataset is annotated with facial landmarks, beauty scores and a beauty score distribution that allow different computational models with different facial beauty prediction paradigms, such as appearance-based and shape-based facial beauty classification, regression and ranking.

This year we provided our beauty perception survey both in a European university as well as in a university in Asia. Currently, we are collecting both the annotator's age as shown in Figure 8 and the gender which is presented in Figure 9.

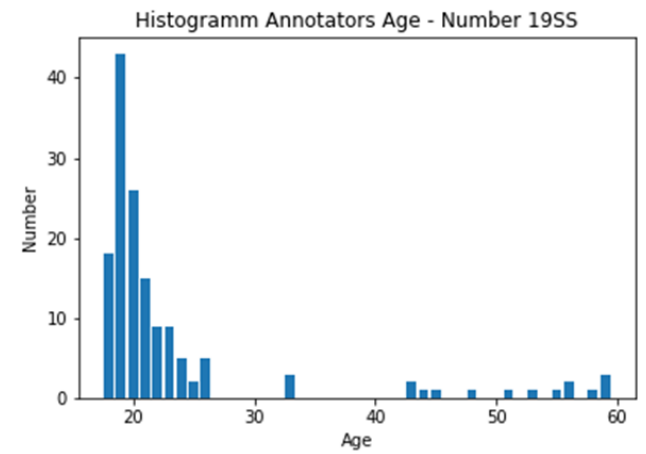

Figure 8: Annotators age distribution.

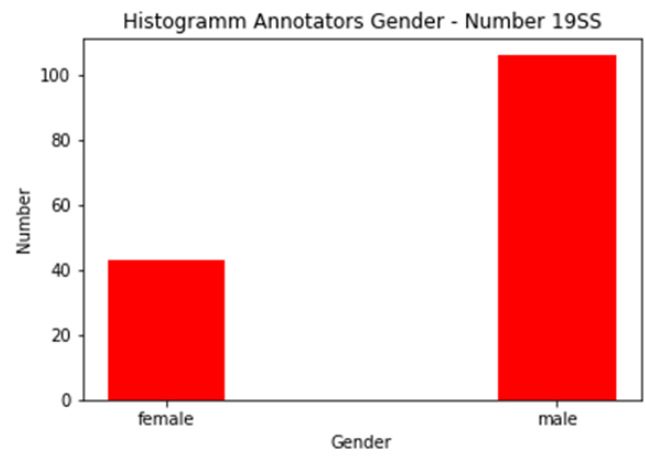

Figure 9: Annotators gender distribution.

To compare the results of surveys in two different cultures we created rankings for the images separately. Our hypothesis was that the average Asian attractiveness should be higher than Caucasian according to the Chinese annotators' results, which is confirmed by Figure 10. Asian annotators think that Asian females are more attractive than Caucasian. To follow this logic, the European annotators result should be the opposite. As we see in Figure 11, the average attractiveness of Caucasian females is now higher. And this is again proof that attractiveness depends a lot on cultures. 


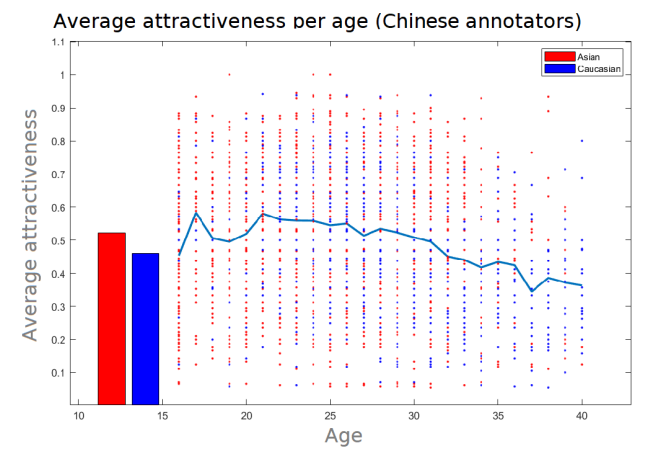

Figure 10: Chinese annotations - attractiveness per age.

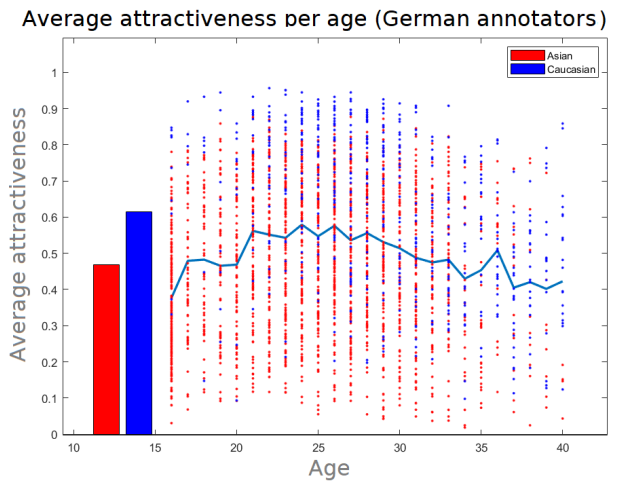

Figure 11: German annotations - attractiveness per age.

\section{EXPERIMENTS AND SOCIAL ETHICS ASPECTS}

\subsection{Female-Face-network (F-net)}

To achieve initial results on aesthetic estimation, we trained the labelled aesthetic dataset using our own F-net network model for face image aesthetic classification. The network model structure is shown in Figure 12.

The F-net network model consists of four convolutional layers, four pooling layers, and two fully connected layers. The model uses the ReLU activation function to add local response normalised LRN operations. The maximum pooling operation is used in each pooling layer. At the same time, F-net uses the same filling method to ensure that image edge information is not lost. The model has the highest recognition accuracy in the Female-Face-dataset, reaching $73 \%$. F-net has a $7 \%$ improvement in recognition accuracy compared to the LeNet-5 model, a 15\% improvement in AlexNet recognition accuracy, and a 9\% improvement in VGG-16 recognition accuracy. The F-net network model is compared with the classical Convolutional Neural Network models LeNet-5, AlexNet, and VGG-16 in the Female-Face-dataset.

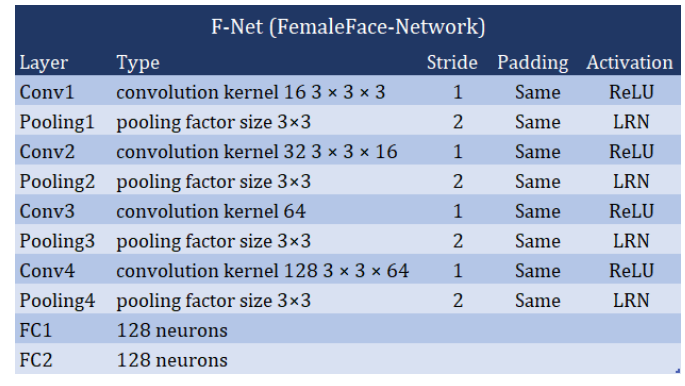

Figure 12: Structure of Female-Face-network model.

\subsection{Network Model Analysis}

We trained the labelled aesthetic dataset on LeNet5, AlexNet and VGG-16. LeNet-5 consists of two convolutional layers, two pooling layers and two fully connected layers. We achieved a relatively high classification recognition accuracy of $66 \%$. AlexNet's classification accuracy in the Female-Face-dataset is only $58 \%$. The accuracy of classification and recognition of test sets is $62 \%$ for VGG- 16 .

\subsection{Different Cultures Social Impact on Aesthetic}

How can a beautiful face be defined? Different cultures have different answers (Larglois et al., 2000; Rhodes et al., 2001). Machine learning is to a certain extent the embodiment of human psychological behaviour and cultural differences lead to a different standard of aesthetics, just as stated in Darwin's (1871) observation that "It is certainly not true that there is in the mind of man any universal standard of beauty with respect to the human body" (Cunningham et al., 1995)

This study reflects the different aesthetic standards for female faces in Asia and Europe. It should be noted that human-like biases observed in ML algorithms have numerous harmful effects, and there exists a growing need to regulate and correct these biases (Fuchs, 2018). From racist Twitter bots (Munger, 2017) to unfortunate Google search results (StephensDavidowitz, 2014), deep-learning software easily picks up on biases. AI-driven decision-making can lead to discrimination in several ways (Borgesius, 2018). Joanna Bryson, a computer scientist at the University of Bath and a co-author of a human-liked bias researching, said: "A lot of people are saying this is showing that AI is prejudiced. No. This is showing we're prejudiced and that $\mathrm{AI}$ is learning it." (Aylin Caliskan, 2017) In this study, we attach great importance to avoid machine learning bias on aesthetic judgment. 
Some researches show that glasses cause a negative effect on ratings of social appearance. The studies from (Terry and Kroger, 1976) and (Thornton, 1944) showed that people with glasses were judged to be less attractive than those without glasses, and a statistically significant effect was found $(p<0.05)$. Figure 7 demonstrates experiments on the AF-dateset across different cultures, subjects and annotators. We can show that humans with glasses are older on average and therefore the glass-wearer-ratings become reasonable based on the demonstrated correlation of aesthetics and age as shown in in Figure 10 and Figure 11.

\section{CONCLUSION}

Confucius has never seen one who loves virtue as much as he loves beauty (Confucius, 1999). The reader of the paper has seen that aesthetics is complex correlated to culture. The Aesthetic-Faces-dataset can be used to quantify this effect by classifying face images and prove hypothesis on a new level. We provide a huge database with many labels and a toolbox with useful AI regression approaches for further work and the research community in machine learning, aesthetics and even social ethics. We want to propose and use 3D Morphable Model face shape analyse and synthesise technologies combined with research with conventional and innovative Convolutional Regression Networks and Extreme Gradient Boosting. The next step is to publish our database on GitLab. After that, the machine learning and face modelling algorithms will be tested, evaluated and published as tool box on GitLab too.

\section{ACKNOWLEDGEMENTS}

This work is partially supported by a grant of the BMBF FHprofUnt program, no. 03FH049PX5

\section{REFERENCES}

Aharon, I., Etcoff, N., Ariely, D., Chabris, C., O'Connor, E., and Breiter, H. C. (2001). Beautiful Faces Have Variable Reward Value: fMRI and Behavioral Evidence. Neuron.

Anderson, S., Adams, G., and Plaut, V. (2008). The cultural grounding of personal relationship: The importance of attractiveness in everyday life. Journal of personality and social psychology, 95:352-68.
Aylin Caliskan, Joanna J. Bryson, A. N. (2017). Semantics derived automatically from language corpora contain human-like biases. Science.

Borgesius, F. Z. (2018). Discrimination, artificial intelligence, and algorithmic decision-making. Directorate General of Democracy.

Chen, T. and Guestrin, C. (2016). Xgboost: A scalable tree boosting system. CoRR, abs/1603.02754.

Confucius (1999). The analects of confucius: a philosophical translation. New York :Ballantine Books, page 9:18.

Cunningham, M., Roberts, A., Barbee, A., Perri, D., and Wu, C.-H. (1995). Their Ideas of Beauty Are, On the Whole, the Same as Ours. Journal of Personality and Social Psychology.

Deffenbacher, K., Vetter, T., Johanson, J., and O'Toole, A. (1998). Facial aging, attractiveness, and distinctiveness. Perception, 27:1233-43.

Eisenthal, Y., Dror, G., and Ruppin, E. (2006). Facial attractiveness: Beauty and the machine. Neural computation, 18:119-42.

Fei, W., Xinjuan, Z., Xiaojun, W., and Rätsch, M. (2019). Aesthetic evaluation of face image based on convolutional neural network.

Friedman, J. (1999). Greedy function approximation: A gradient boosting machine. The Annals of Statistics, 29.

Fuchs, D. J. (2018). The Dangers of Human-Like Bias in Machine-Learning Algorithms. Missouri S\&T's Peer to Peer2.

Henss, R. (2006). Perceiving age and attractiveness in facial photographs. Journal of Applied Social Psychology, 21:933 - 946.

Huang, G. B., Ramesh, M., Berg, T., and Learned-Miller, E. (2007). Labeled faces in the wild: A database for studying face recognition in unconstrained environments. Technical Report 07-49, University of Massachusetts, Amherst.

Hyland, D. (2008). Plato and the Question of Beauty. Studies in continental thought. https://books.google.de/books?id=C1LaszjLNawC.

International Olympic Committee (Retrieved 03/08/2013). Olympic Photos, Galleries and Slideshows,. https://www.olympic.org/photos.

Jones, F., Harris, P., and Chrispin, C. (2000). Catching the sun: An investigation of sun-exposure and skin protective behaviour. Psychology Health \& Medicine PSYCHOL HEALTH MED, 5:131-141.

Kelly, M. (1998). Encyclopedia of Aesthetics. Oxford University Press.

Langlois, J., Kalakanis, L., Rubenstein, A., Larson, A., Hallam, M., and Smoot, M. (2000). Maxims or myths of beauty? a meta-analytic and theoretical review. Psychological bulletin, 126:390-423.

Larglois, J., Kalakanis, L., Rubenstein, A., Larson, A., Hallam, M., and Smoot, M. (2000). Maxims or myths of beauty? A meta-analytic and theoretical review. Psychol. Bull. 
Lehmann, R. and Wohlrabe, K. (2017). Who is the 'journal grand master'? a new ranking based on the elo rating system. Journal of Informetrics, 11(3):800 - 809.

Li, E. P., Min, H. J., Belk, R. W., Kimura, J., and Bahl, S. (2008). Skin lightening and beauty in four asian cultures. Advances in Consumer Research, 35:444449.

Liang, L., Lin, L., Jin, L., Xie, D., and Li, M. (2018). Scut-fbp5500: A diverse benchmark dataset for multiparadigm facial beauty prediction.

Little, A. and Roberts, S. (2012). Evolution, appearance, and occupational success. Evolutionary psychology: an international journal of evolutionary approaches to psychology and behavior, 10:782-801.

Mazis, M., Ringold, D., Perry, E., and Denman, D. (1992). Perceived age and attractiveness of models in cigarette advertisements. Journal of Marketing, 56.

Munger, K. P. B. (2017). Tweetment Effects on the Tweeted: Experimentally Reducing Racist Harassment. Springer.

Porter, J. I. (2017). Beauty, Value, and the Aesthetics of Life in Kant and Aristotle. Republics of Letters, Volume 5, Issue 1. University of California, Berkeley.

Redi, M., Rasiwasia, N., Aggarwal, G., and Jaimes, A. (2015). The beauty of capturing faces: Rating the quality of digital portraits.

Rhodes, G., Yoshikawa, S., Clark, A., Lee, K., McKay, R., and Akamatsu, S. (2001). Attractiveness of facial averageness and symmetry in non-Western cultures: in search of biologically based standards of beauty. Perception.

Rosenblatt, F. (1958). The perceptron: A probabilistic model for information storage and organization in the brain. Psychological Review, pages 65-386.

Russakovsky, O., Deng, J., Su, H., Krause, J., Satheesh, S., Ma, S., Huang, Z., Karpathy, A., Khosla, A., Bernstein, M. S., Berg, A. C., and Li, F. (2015). Imagenet large scale visual recognition challenge. International Journal of Computer Vision, 115(3):211-252.

Sahay, S. and Piran, N. (1997). Skin-color preferences and body satisfaction among south asian-canadian and european-canadian female university students. The Journal of Social Psychology, 137(2):161-171. PMID: 9140216.

Salzborn, S. and Stich, T. (2016). Samuel P. Huntington: The Clash of Civilizations and the Remaking of World Order, New York: Simon \& Schuster 1996, 368 S. (dt. Kampf der Kulturen. Die Neugestaltung der Weltpolitik im 21. Jahrhundert, München/Wien: Europa Verlag 1996, 584 S.), pages 401-403. Springer Fachmedien Wiesbaden, Wiesbaden.

Stephens-Davidowitz, S. (2014). The cost of racial animus on a black candidate: Evidence using Google search data. Journal of Public Economics.

Sturm, A., Brissett, A., and Brissett, A. (2010). Ethnic trends in facial plastic surgery. Facial plastic surgery :FPS, 26:69-74.

Terry, R. L. and Kroger, D. L. (1976). Effects of eye correctives on ratings of attractiveness. Perceptual and Motor Skills.
Thornton, G. R. (1944). The effect of wearing glasses upon judgments of personality traits of persons seen briefly. Journal of Applied Psychology.

van der Geld, P., Oosterveld, P., van Heck, G., and Kuijpers-Jagtman, A. (2007). Smile attractiveness. self-perception and influence on personality. Angle Orthodontist, 77(5):759-765.

Wang, L. (2016). On confucius's ideology of aesthetic order.

Xie, D., Liang, L., Jin, L., Xu, J., and Li, M. (2015). SCUTFBP: A benchmark dataset for facial beauty perception. In $S M C$, pages 1821-1826. IEEE.

Xie, Q. V. and Zhang, M. (2013). White or tan? a crosscultural analysis of skin beauty advertisements between china and the united states. Asian Journal of Communication, 23(5):538-554.

Yuanchong, X. (1993). Book of Poetry. Changsha: Hunan Publishing House. 